\title{
Dissipative Particle Dynamics Simulation of Self-Assembly in a Bolaamphiphilic Solution*)
}

\author{
Susumu FUJIWARA, Yu TAKAHASHI, Hiroki IKEBE, Tomoko MIZUGUCHI, \\ Masato HASHIMOTO, Yuichi TAMURA ${ }^{1)}$, Hiroaki NAKAMURA ${ }^{2)}$ and Ritoku HORIUCHI ${ }^{2)}$ \\ Kyoto Institute of Technology, Matsugasaki, Sakyo-ku, Kyoto 606-8585, Japan \\ ${ }^{1)}$ Konan University, 8-9-1 Okamoto, Higashinada-ku, Kobe 658-8501, Japan \\ 2) National Institute for Fusion Science, 322-6 Oroshi-cho, Toki 509-5292, Japan
}

(Received 1 December 2015 / Accepted 24 February 2016)

\begin{abstract}
The self-assembly of flexible bolaamphiphilic molecules in a solution is studied by dissipative particle dynamics simulations. In particular, we investigate the effect of the interaction difference, $\Delta a$, between the two different hydrophilic end groups on the self-assembly in a bolaamphiphilic solution. Our simulations show that two types of self-assembled structures, spherical vesicles and worm-like micelles, are obtained from a random configuration of bolaamphiphilic molecules in a solution. We find that the worm-like micelles are formed when $\Delta a>0$, whereas spherical vesicles are obtained when $\Delta a \leq 0$. It is also ascertained that the size of the spherical vesicles decreases as $\Delta a$ decreases.
\end{abstract}

(c) 2016 The Japan Society of Plasma Science and Nuclear Fusion Research

Keywords: dissipative particle dynamics simulation, self-assembly, bolaamphiphilic solution, worm-like micelle, spherical vesicle

DOI: $10.1585 /$ pfr.11.2401073

\section{Introduction}

Recently, spontaneous formation of structures has gained considerable interest in nonequilibrium and nonlinear systems, including plasma systems and soft matter systems. We aim at improving plasma confinement by clarifying the self-organizing properties common to these systems. To this end, we investigate the structure formation in soft matter systems, e.g., the formation of micelles and mesophases in amphiphilic molecular systems [1-9] and the formation of orientationally ordered structures in polymeric systems [10-15]. Amphiphilic molecules, such as surfactants and lipids, comprise both hydrophilic and hydrophobic groups. In aqueous solvents, they spontaneously self-assemble into various structures such as micelles, lamellar structures, and vesicles [16-18]. Numerous computer simulation studies have been conducted on the structure formation of amphiphilic molecules, each of which is composed of a hydrophilic head group and a hydrophobic tail group. In contrast, few theoretical and simulation studies have been carried out on the structure formation of bolaamphiphilic molecules, each of which consists of a hydrophobic stalk group and two hydrophilic end groups. Although a few molecular simulation studies have been performed to investigate the phase behavior of a bolaamphiphilic solution $[9,19]$ and the shape transformations of vesicles formed by amphiphilic triblock copolymers [20], little is known about the detailed self-assembly

author's e-mail: fujiwara@ kit.ac.jp

*) This article is based on the presentation at the 25th International Toki Conference (ITC25). processes in a bolaamphiphilic solution at the molecular level.

The purpose of this study is to determine the effect of the interaction difference between the two different hydrophilic end groups on the self-assembly in a bolaamphiphilic solution. To investigate the self-assembly in a bolaamphiphilic solution at the molecular level, we perform dissipative particle dynamics (DPD) simulations of a bolaamphiphilic solution and analyze the formation processes of micelles and vesicles.

\section{Simulation Model and Method}

The computational model used is similar to the one used by Li et al. [20]. A bolaamphiphilic molecule is represented by a coarse-grained flexible chain, $\mathrm{AB}_{3} \mathrm{C}$, comprising a hydrophobic stalk with three particles (denoted by $\mathrm{B}$ ) and two hydrophilic ends (denoted by A and C), each of which consists of one particle. A solvent molecule is modeled as a hydrophilic particle (denoted by S). The mass of each particle is assumed to be unity. The total force on particle $i$ is written as the sum of four components, a conservative force $\mathbf{F}_{i j}^{\mathrm{C}}$, a dissipative force $\mathbf{F}_{i j}^{\mathrm{D}}$, a random force $\mathbf{F}_{i j}^{\mathrm{R}}$, and a harmonic spring force $\mathbf{F}_{i j}^{\mathrm{S}}$, to represent the bond stretching between the bonded neighboring particles:

$$
\mathbf{F}_{i}=\sum_{j \neq i}\left(\mathbf{F}_{i j}^{\mathrm{C}}+\mathbf{F}_{i j}^{\mathrm{D}}+\mathbf{F}_{i j}^{\mathrm{R}}+\mathbf{F}_{i j}^{\mathrm{S}}\right) .
$$

The conservative force is a soft repulsion of the form

$$
\mathbf{F}_{i j}^{\mathrm{C}}=\left\{\begin{array}{cc}
a_{i j}\left(1-r_{i j}\right) \hat{\mathbf{r}}_{i j} & \left(r_{i j}<1\right) \\
0 & \left(r_{i j} \geq 1\right)
\end{array} .\right.
$$


Here, $a_{i j}$ is the maximum repulsion between $i$ and $j$, and $\mathbf{r}_{i j}=\mathbf{r}_{i}-\mathbf{r}_{j}, r_{i j}=\left|\mathbf{r}_{i j}\right|, \hat{\mathbf{r}}_{i j}=\mathbf{r}_{i j} / r_{i j}$, where $\mathbf{r}_{i}$ is a position vector of particle $i$. The dissipative (or drag) force and the random force are, respectively, given by

$$
\mathbf{F}_{i j}^{\mathrm{D}}=-\gamma w^{\mathrm{D}}\left(r_{i j}\right)\left(\hat{\mathbf{r}}_{i j} \cdot \mathbf{v}_{i j}\right) \hat{\mathbf{r}}_{i j}
$$

and

$$
\mathbf{F}_{i j}^{\mathrm{R}}=\sigma w^{\mathrm{R}}\left(r_{i j}\right) \theta_{i j} \hat{\mathbf{r}}_{i j}
$$

Here, $\gamma$ and $\sigma$ are the friction coefficient and the noise amplitude, respectively; $w^{\mathrm{D}}$ and $w^{\mathrm{R}}$ are the weight functions, which provide the range of interaction for the dissipative and random forces; $\theta_{i j}(t)$ is a randomly fluctuating variable with Gaussian statistics: $\left\langle\theta_{i j}(t)\right\rangle=0$ and $\left\langle\theta_{i j}(t) \theta_{k l}\left(t^{\prime}\right)\right\rangle=\left(\delta_{i k} \delta_{j l}+\delta_{i l} \delta_{j k}\right) \delta\left(t-t^{\prime}\right)$; and $\mathbf{v}_{i j}=\mathbf{v}_{i}-\mathbf{v}_{j}$, where $\mathbf{v}_{i}$ is a velocity vector of particle $i$. According to Español and Warren [21], the equilibrium distribution of the system becomes the Gibbs-Boltzmann distribution if the weight functions $\left(w^{\mathrm{D}}\right.$ and $\left.w^{\mathrm{R}}\right)$ and the coefficients of the dissipation and the random forces $(\gamma$ and $\sigma$ ) satisfy the following conditions:

$$
\begin{aligned}
& w^{\mathrm{D}}\left(r_{i j}\right)=\left[w^{\mathrm{R}}\left(r_{i j}\right)\right]^{2}=\left\{\begin{array}{cc}
(1-r)^{2} & (r<1) \\
0 & (r \geq 1)
\end{array},\right. \\
& \sigma^{2}=2 \gamma k_{\mathrm{B}} T .
\end{aligned}
$$

This is the fluctuation-dissipation theorem for the DPD method. The values $\sigma=2.4$ and $\gamma=4.5$ are used in our simulation, and the temperature of the system is maintained at $k_{\mathrm{B}} T=0.64$, where $k_{\mathrm{B}}$ is the Boltzmann constant. The harmonic spring force for the bond stretching between the bonded neighboring particles is given by

$$
\mathbf{F}_{i j}^{\mathrm{S}}=k_{\mathrm{s}}\left(1-r_{i j} / r_{\mathrm{s}}\right) \hat{\mathbf{r}}_{i j},
$$

where $k_{\mathrm{s}}$ is the spring constant and $r_{\mathrm{s}}$ is the equilibrium bond length. In our simulation, we use $k_{\mathrm{s}}=10.0$ and $r_{\mathrm{s}}=$ 0.86 .

A modified version of the velocity-Verlet algorithm [22] is used to advance the set of positions and velocities:

$$
\begin{aligned}
& \mathbf{r}_{i}(t+\Delta t)=\mathbf{r}_{i}(t)+\Delta t \mathbf{v}_{i}(t)+\frac{1}{2}(\Delta t)^{2} \mathbf{F}_{i}(t), \\
& \tilde{\mathbf{v}}_{i}(t+\Delta t)=\mathbf{v}_{i}(t)+\lambda \Delta t \mathbf{F}_{i}(t), \\
& \mathbf{F}_{i}(t+\Delta t)=\mathbf{F}_{i}(\mathbf{r}(t+\Delta t), \tilde{\mathbf{v}}(t+\Delta t)), \\
& \mathbf{v}_{i}(t+\Delta t)=\mathbf{v}_{i}(t)+\frac{1}{2} \Delta t\left(\mathbf{F}_{i}(t)+\mathbf{F}_{i}(t+\Delta t)\right),
\end{aligned}
$$

where $\Delta t$ is a time step and $\lambda$ is a variable factor. Note that the actual velocity-Verlet algorithm would be recovered for $\lambda=1 / 2$, if the force did not depend on velocity. Using the time step $\Delta t$, the random force can be rewritten as

$$
\mathbf{F}_{i j}^{\mathrm{R}}=\sigma w^{\mathrm{R}}\left(r_{i j}\right) \varsigma_{i j} \Delta t^{-1 / 2} \hat{\mathbf{r}}_{i j},
$$

where $\varsigma_{i j}$ is a random variable with zero mean and unit variance. The appearance of $\Delta t^{-1 / 2}$ in Eq. (9) can be derived by integrating the underlying stochastic differential equations and interpreting the random force as a Wiener process [21]. The heuristic argument as to why $\Delta t^{-1 / 2}$ appears can be seen in Ref. [23].

The DPD simulations with $\lambda=0.65$ and $\Delta t=0.04$ are performed using COGNAC in the OCTA system [24]. We apply periodic boundary conditions and the number density is set at $\rho=3.0$. The repulsive interaction parameters, $a_{i j}$, in Eq. (2) are given by

$$
a_{i j}=\left(\begin{array}{ccccc} 
& \mathrm{A} & \mathrm{B} & \mathrm{C} & \mathrm{S} \\
\mathrm{A} & a_{\mathrm{AA}} & 200 & 200 & 25 \\
\mathrm{~B} & 200 & 25 & 200 & 200 \\
\mathrm{C} & 200 & 200 & 30 & 25 \\
\mathrm{~S} & 25 & 200 & 25 & 25
\end{array}\right) \text {, }
$$

where $a_{\mathrm{AA}}$ varies from 20 to 50. In this study, we discuss the self-assembled structures obtained by our DPD simulation from the viewpoint of the curvature of the hydrophobic stalk-solvent interface, which is related to the interaction difference of the two hydrophilic ends, $\Delta a \equiv a_{\mathrm{AA}}-a_{\mathrm{CC}}$. The effect of $\Delta a$ on the interfacial curvature for a fixed value of $a_{\mathrm{CC}}$ is illustrated in Fig. 1.

The simulations are initiated from a random configuration of 1000 bolaamphiphilic molecules, $\mathrm{AB}_{3} \mathrm{C}$, and 40000 solvent molecules, $\mathrm{S}$, in a $24.66 \times 24.66 \times 24.66$ cubic box. The total number of particles is 45000 . We perform the DPD simulations of $t=4.0 \times 10^{4}\left(1.0 \times 10^{6}\right.$ time steps) for each simulation run and analyze the selfassembly processes for various values of the interaction difference between the two hydrophilic end groups, $\Delta a \equiv$ $a_{\mathrm{AA}}-a_{\mathrm{CC}}=a_{\mathrm{AA}}-30(-10 \leq \Delta a \leq 30)$. (a) $\Delta a<0$

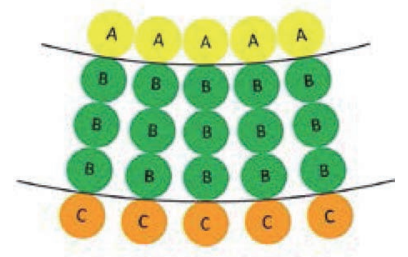

(b) $\Delta a=0$

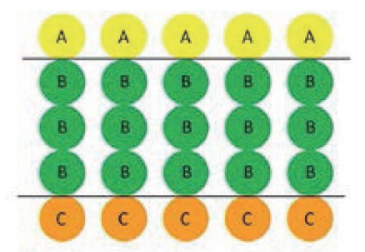

(c) $\Delta a>0$

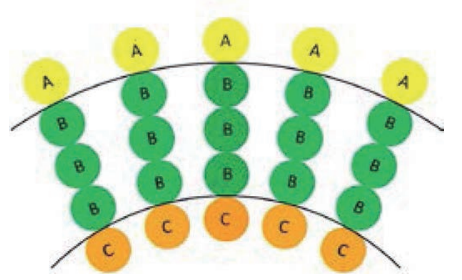

Fig. 1 Schematic of the effects of the interaction difference of the two hydrophilic ends, $\Delta a\left(\equiv a_{\mathrm{AA}}-a_{\mathrm{CC}}\right)$, on the interfacial curvature for a fixed value of $a_{\mathrm{CC}}$ : (a) $\Delta a<0$, (b) $\Delta a=0$, and (c) $\Delta a>0$. Yellow, orange and green particles denote the hydrophilic particles (A and C) and the hydrophobic particles (B), respectively. The curvature of the hydrophobic stalk-solvent interface (thick line) changes sign at $\Delta a=0$. 
(a) $t=0$

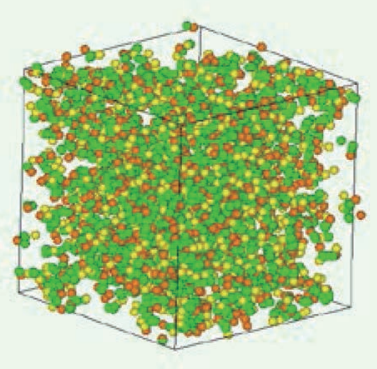

(c) $t=40000$

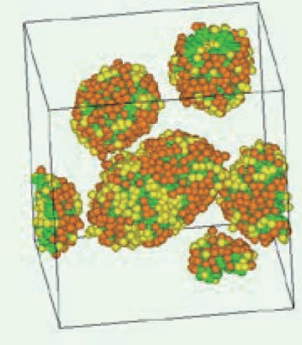

(b) $t=4000$

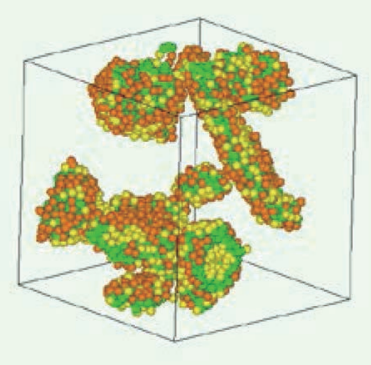

(d) $t=40000$

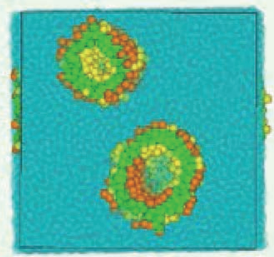

(a) $t=0$

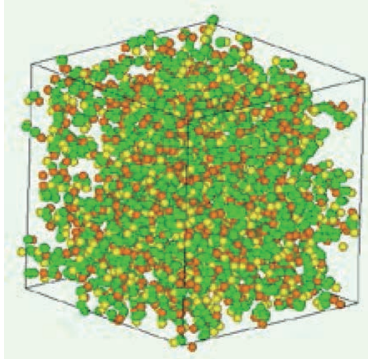

(c) $t=40000$

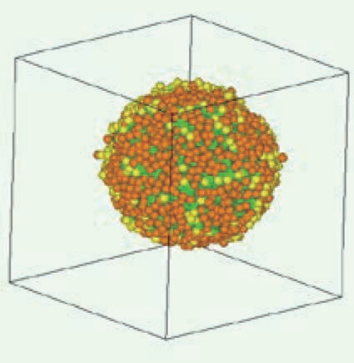

(b) $t=10000$

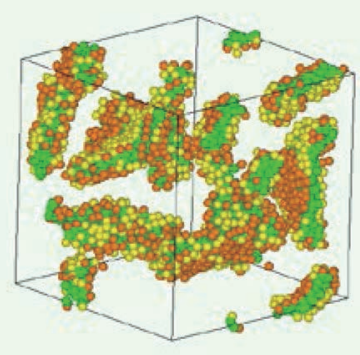

(d) $t=40000$

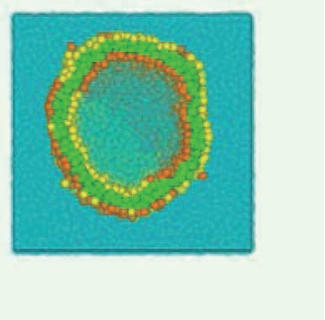

Fig. 2 Images of the self-assembly process for $a_{\mathrm{AA}}=20(\Delta a=$ -10): (a) $t=0$, (b) $t=4000$, and (c), (d) $t=40000$. Yellow and orange particles denote the hydrophilic particles, whereas green particles denote the hydrophobic particles. Solvent molecules are not displayed in (a) - (c), whereas in (d), they are represented by transparent light blue particles.

\section{Simulation Results and Discussion 3.1 Formation of self-assembled structures}

In order to demonstrate the formation processes of the self-assembled structures, we present images of the spontaneous aggregation process at various times for $a_{\mathrm{AA}}=20$ $(\Delta a=-10), a_{\mathrm{AA}}=30(\Delta a=0)$, and $a_{\mathrm{AA}}=40(\Delta a=10)$, in Figs. $2-4$, respectively. The following characteristic features are observed from these figures. (i) Spherical vesicles are obtained at $\Delta a \leq 0$, whereas worm-like micelles are formed at $\Delta a>0$. (ii) The size of the spherical vesicles decreases as $\Delta a$ decreases. (iii) In the case of $\Delta a=0$, the configuration of the bolaamphiphilic molecules is random at the initial time (Fig. 3(a)). As time elapses, small disc micelles are formed at several positions (Fig. 3 (b)). Several small disc micelles then coalesce into a large micelle, and the spherical vesicle is finally formed (Figs. 3 (c) and (d)). As illustrated in Fig. 1, the curvature of the hydrophobic stalk-solvent interface tends to zero, when $\Delta a=0$. Therefore, for $\Delta a=0$, the disc micelles and the spherical vesicles are observed rather than the worm-like micelles.

\subsection{Potential energy}

Figures 5-7 show the total potential energy $E_{\text {pot }}$ and the number of clusters $n_{\mathrm{c}}$ for $a_{\mathrm{AA}}=20(\Delta a=-10)$, $a_{\mathrm{AA}}=30(\Delta a=0)$, and $a_{\mathrm{AA}}=40(\Delta a=10)$, respectively. We observe the following features from these figures. (i) The total potential energy decreases with decreasing num-
Fig. 3 Images of the self-assembly process for $a_{\mathrm{AA}}=30(\Delta a=$ 0 ): (a) $t=0$, (b) $t=10000$, and (c), (d) $t=40000$. Yellow and orange particles denote the hydrophilic particles, whereas green particles denote the hydrophobic particles. Solvent molecules are not displayed in (a) - (c), whereas in (d), they are represented by transparent light blue particles. (a) $t=0$

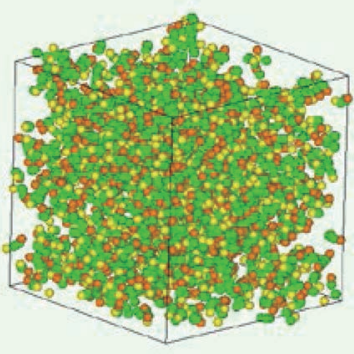

(c) $t=40000$

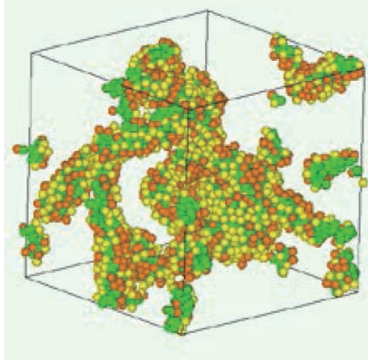

(b) $t=10000$

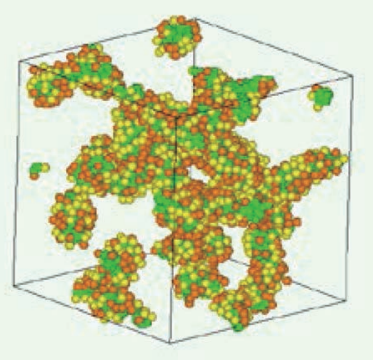

(d) $t=40000$

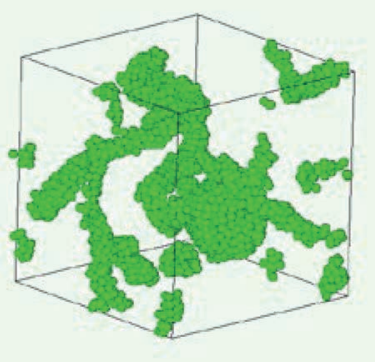

Fig. 4 Images of the self-assembly process for $a_{\mathrm{AA}}=40(\Delta a=$ 10): (a) $t=0$, (b) $t=10000$, and (c), (d) $t=40000$. Yellow and orange particles denote the hydrophilic particles, whereas green particles denote the hydrophobic particles. Only hydrophobic particles (green) are shown in (d). Solvent molecules are not displayed. 

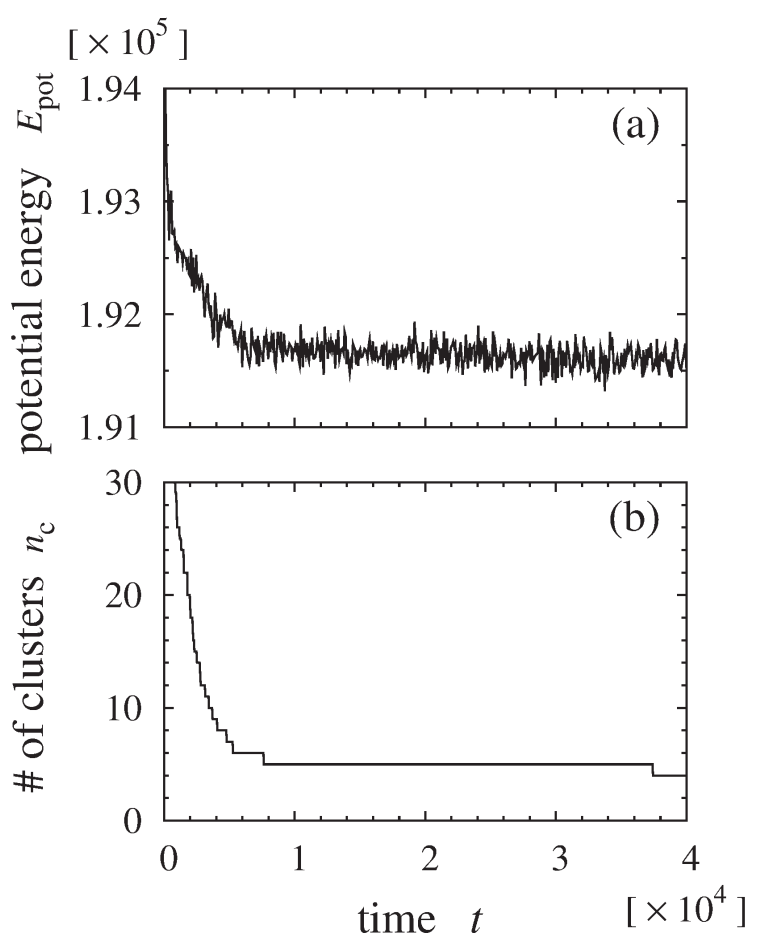

Fig. 5 The time evolution of (a) the total potential energy $E_{\text {pot }}$ and (b) the number of clusters $n_{\mathrm{c}}$ for $a_{\mathrm{AA}}=20(\Delta a=$ $-10)$.
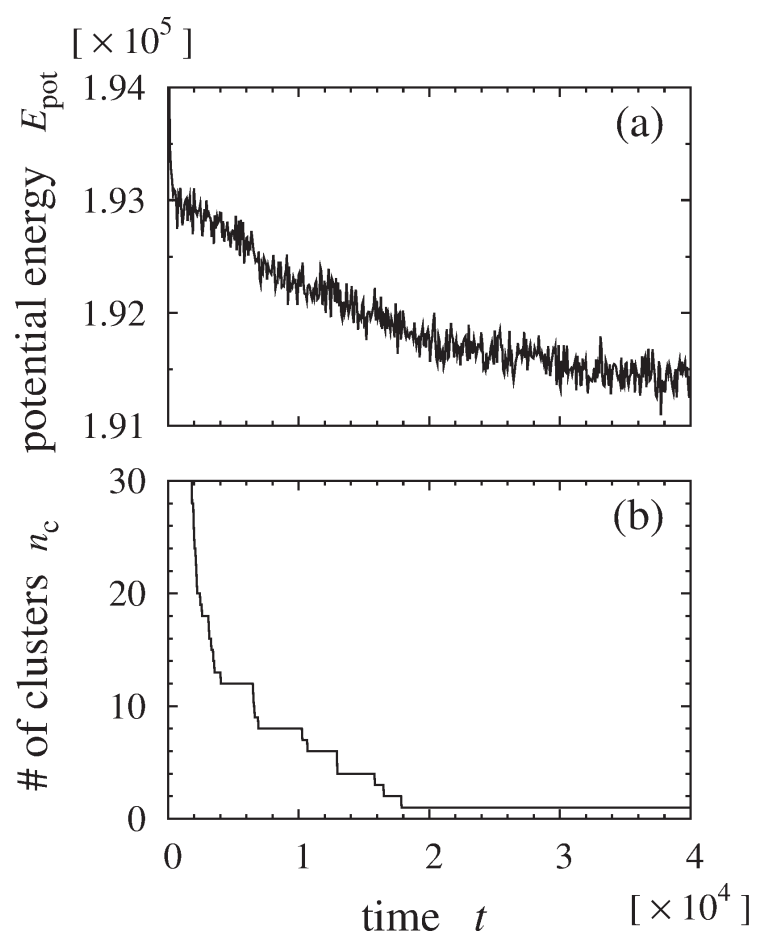

Fig. 6 The time evolution of (a) the total potential energy $E_{\text {pot }}$ and (b) the number of clusters $n_{\mathrm{c}}$ for $a_{\mathrm{AA}}=30(\Delta a=0)$.

ber of clusters (or growth of clusters). (ii) The total potential energy of the spherical vesicles (Figs. 5 (a) and 6 (a)) is lower than that of the worm-like micelles (Fig. 7 (a)). (iii) Small vesicles are rapidly formed (Fig. 6(a)) but the for-

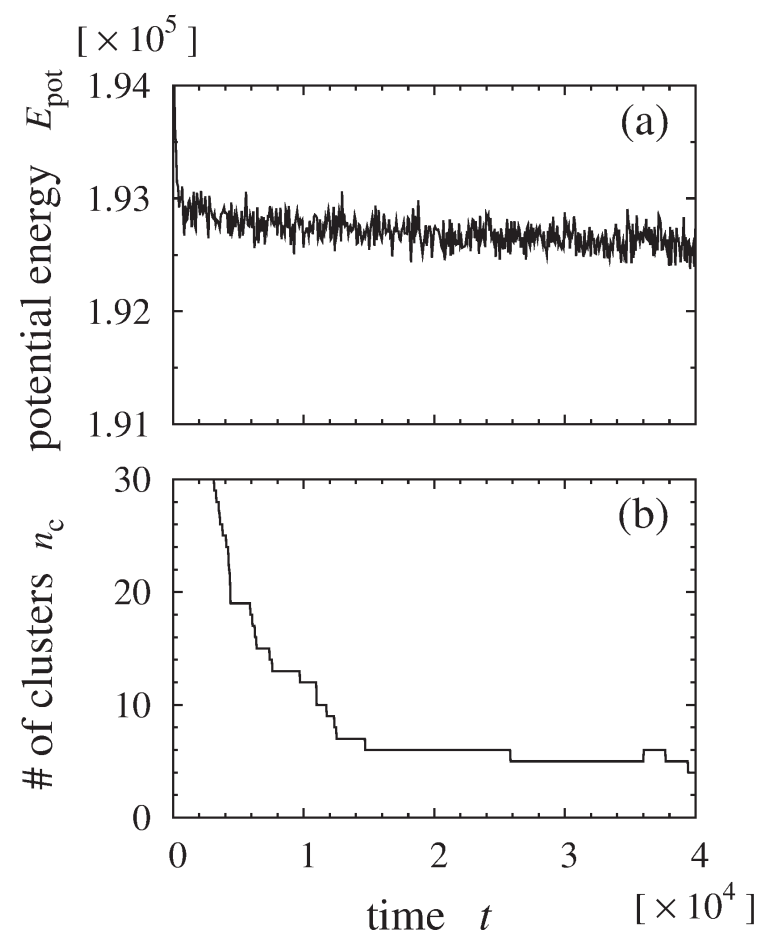

Fig. 7 The time evolution of (a) the total potential energy $E_{\text {pot }}$ and (b) the number of clusters $n_{\mathrm{c}}$ for $a_{\mathrm{AA}}=40(\Delta a=10)$.

mation of large vesicles takes a long time (Fig. 6(b)).

\subsection{Chain conformation}

The chain conformation is examined to elucidate the internal structures of the spherical vesicles and the wormlike micelles. In Fig. 8, we show the distribution of the bond angle $P(\theta)$ for $a_{\mathrm{AA}}=20(\Delta a=-10), a_{\mathrm{AA}}=30$ $(\Delta a=0)$, and $a_{\mathrm{AA}}=40(\Delta a=10)$. The bond angles $\theta_{1}$, $\theta_{2}$, and $\theta_{3}$ represent the angles $\angle \mathrm{ABB}, \angle \mathrm{BBB}$, and $\angle \mathrm{BBC}$, respectively. For all values of $a_{\mathrm{AA}}$ (or $\left.\Delta a\right), P\left(\theta_{1}\right)$ and $P\left(\theta_{3}\right)$ exhibit a peak at $\theta_{1}, \theta_{3}<\pi / 3$, whereas $P\left(\theta_{2}\right)$ presents a peak at $\theta_{2}>\pi / 3$. The peak positions of $P\left(\theta_{1}\right)$ and $P\left(\theta_{3}\right)$ are the same, when $a_{\mathrm{AA}}=30(\Delta a=0)$, due to the fact that the two types of hydrophilic particles, $\mathrm{A}$ and $\mathrm{C}$, are indistinguishable from each other. It is also found that as $a_{\mathrm{AA}}$ (or $\Delta a$ ) increases, the peak position of $P\left(\theta_{1}\right)$ decreases, whereas those of $P\left(\theta_{2}\right)$ and $P\left(\theta_{3}\right)$ increase.

\section{Conclusions}

In this study, we carried out dissipative particle dynamics simulations of coarse-grained bolaamphiphilic molecules in a solution. By analyzing the self-assembly processes of bolaamphiphilic molecules, the following results were obtained: (1) Two types of self-assembled structures (spherical vesicles and worm-like micelles) are formed from a random configuration of bolaamphiphilic molecules in a solution. (2) Worm-like micelles are obtained when the interaction difference, $\Delta a$, between the two different hydrophilic ends is positive $(\Delta a>0)$, whereas 


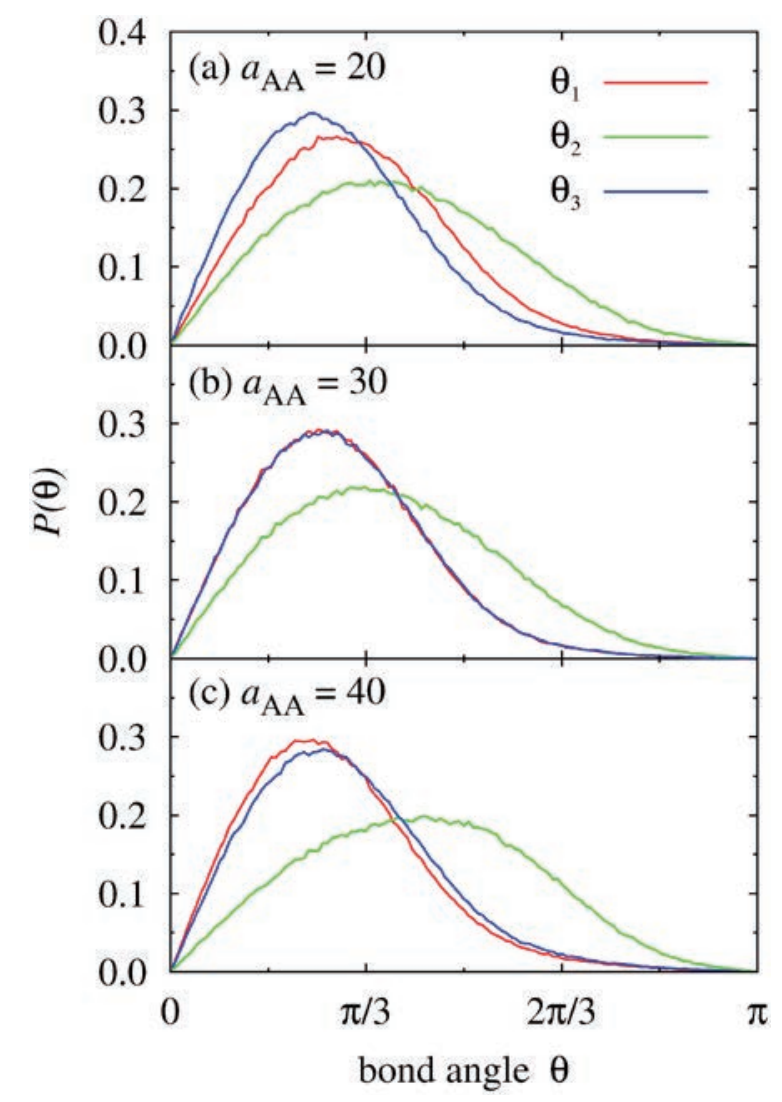

Fig. 8 Distribution function of the bond angle $P(\theta)$ (a) for $a_{\mathrm{AA}}=$ $20(\Delta a=-10)$, (b) for $a_{\mathrm{AA}}=30(\Delta a=0)$, and (c) for $a_{\mathrm{AA}}=40(\Delta a=10)$. The bond angles $\theta_{1}, \theta_{2}$, and $\theta_{3}$ correspond to the angles $\angle \mathrm{ABB}, \angle \mathrm{BBB}$, and $\angle \mathrm{BBC}$.

spherical vesicles are formed when $\Delta a \leq 0$. (3) The size of the spherical vesicles is directly correlated with $\Delta a$.

In this study, we performed DPD simulations under the condition $a_{\mathrm{CC}}=30>a_{\mathrm{SS}}=25$. It would be very interesting to investigate what types of self-assembled structures are formed when $a_{\mathrm{CC}}<a_{\mathrm{SS}}$. In our future study, we will carry out DPD simulations for $a_{\mathrm{CC}}<a_{\mathrm{SS}}$. We will also investigate the formation processes of worm-like micelles and vesicles in a bolaamphiphilic solution by coarsegrained molecular dynamics simulations.

\section{Acknowledgment}

This study was partially supported by the NIFS Collaborative Research Program (NIFS14KNTS035). The authors thank the Supercomputer Center, Institute for Solid State Physics, University of Tokyo, for the facilities provided. The authors would like to thank Enago (www.enago.jp) for the English language review.

[1] S. Fujiwara, M. Hashimoto and T. Itoh, J. Plasma Phys. 72, 1011 (2006).

[2] S. Fujiwara, T. Itoh, M. Hashimoto and Y. Tamura, Mol. Simul. 33, 115 (2007).

[3] S. Fujiwara, T. Itoh, M. Hashimoto and R. Horiuchi, J. Chem. Phys. 130, 144901 (2009).

[4] S. Fujiwara, T. Itoh, M. Hashimoto, H. Nakamura and Y. Tamura, Plasma Fusion Res. 5, S2114 (2010).

[5] S. Fujiwara, T. Itoh, M. Hashimoto, Y. Tamura, H. Nakamura and R. Horiuchi, Plasma Fusion Res. 6, 2401040 (2011).

[6] S. Fujiwara, D. Funaoka, T. Itoh and M. Hashimoto, Comput. Phys. Commun. 182, 192 (2011).

[7] S. Fujiwara, M. Hashimoto, T. Itoh and R. Horiuchi, Chem. Lett. 41, 1038 (2012).

[8] S. Fujiwara, M. Hashimoto, Y. Tamura, H. Nakamura and R. Horiuchi, Plasma Fusion Res. 9, 3401067 (2014).

[9] S. Fujiwara, T. Miyata, M. Hashimoto, Y. Tamura, H. Nakamura and R. Horiuchi, Plasma Fusion Res. 10, 3401029 (2015).

[10] S. Fujiwara and T. Sato, J. Chem. Phys. 107, 613 (1997).

[11] S. Fujiwara and T. Sato, J. Chem. Phys. 114, 6455 (2001).

[12] S. Fujiwara and T. Sato, Phys. Rev. Lett. 80, 991 (1998).

[13] S. Fujiwara and T. Sato, J. Chem. Phys. 110, 9757 (1999).

[14] S. Fujiwara and T. Sato, Comput. Phys. Commun. 142, 123 (2001).

[15] S. Fujiwara, M. Hashimoto, T. Itoh and H. Nakamura, J. Phys. Soc. Jpn. 75, 024605 (2006).

[16] J.N. Israelachvili, Intermolecular and Surface Forces (Academic Press, London, 1992) 2nd ed.

[17] Micelles, Membranes, Microemulsions, and Monolayers, edited by W.M. Gelbart, A. Ben-Shaul and D. Roux (Springer-Verlag, New York, 1994), pp.1-104.

[18] I.W. Hamley, Introduction to Soft Matter (J. Wiley, Chichester, 2007) Rev. ed.

[19] R. Shirasaki, Y. Yoshikai, H. Qian, S. Fujiwara, Y. Tamura and H. Nakamura, Plasma Fusion Res. 6, 2401116 (2011).

[20] X. Li, I.V. Pivkin, H. Liang and G.E. Karniadakis, Macromolecules 42, 3195 (2009).

[21] P. Español and P.B. Warren, Europhys. Lett. 30, 191 (1995).

[22] M.P. Allen and D.J. Tildesley, Computer Simulation of Liquids (Clarendon, Oxford, 1987).

[23] R.D. Groot and P.B. Warren, J. Chem. Phys. 107, 4423 (1997).

[24] http://octa.jp/ 